\title{
СТАБИЛИЗАЦИЯ СИСТЕМЫ СВЯЗАННЫХ ОБРАТНЫХ МАЯТНИКОВ ВЕРТИКАЛЬНЫМ УПРАВЛЕНИЕМ
}

\author{
(c) 2020 М. Е. Семёнов ${ }^{\varpi 1,2,3}$, О. И. Канищева ${ }^{4}$, М. А. Попов ${ }^{3}$ \\ ${ }^{1}$ Федеральный исследовательский иентр «Единая геофизическая служба Российской академии наук» \\ пр. Ленина, 189, 249035 Обнинск, Калужская обл., Российская Федерация \\ ${ }^{2}$ Воронежский государственньй университет \\ Университетская пл., 1, 394018 Воронеж, Российская Федерация \\ ${ }^{3}$ Вороненский государственный технический университет \\ ул. 20-лет Октября, 84, 394064 Воронеж, Российская Федераиия \\ ${ }^{4}$ Военный учебно-научный иентр Военно-воздушных сил «Военно-воздушная академия \\ имени пробессора Н. Е. Жуковского и Ю. А. Гагарина» \\ ул. Старых Большевиков, 54а, 371600Воронеж, Российская Федерация
}

\begin{abstract}
Аннотация. При решении ряда практически важных задач (колебания поддерживающих контуров в строительстве, проблема стабилизации плазмы, стабилизация синтезированных биологических цепочек и т. п.) модели систем основываются на законах движения простейших связанных осцилляторов и их цепочек. В данной статье рассматривается математическая модель системы, состоящей из двух обратных маятников с упругой связью, представленной пружиной. Система управляется посредством применения управляющего воздействия, представляющего собой вертикальные осцилляции точки крепления одного из маятников. Проведено детальное исследование динамики указанной механической системы, сформулированы условия, обеспечивающие ее стабилизацию. Построены зоны устойчивости в пространстве исходных параметров. Представлена эволюция зон устойчивости в зависимости от значений жёсткости пружины. Построены спектры решений, показывающие, что движения системы соответствуют почти периодическим функциям. Установлено наличие неустойчивых периодических режимов на границах зон устойчивости. Определены плоскости, соответствующие начальным условиям, отвечающим найденным периодическим решениям. Основные аналитические результаты получены с использованием матрицы монодромии. В рассматриваемом в работе случае, когда система в линейном приближении является кусочно-линейной, эта матрица может быть представлена в явной форме. В работе также приведены результаты численных экспериментов, иллюстрирующих динамику системы. Также показано, что с изменением естественных параметров системы геометрия зон устойчивости претерпевает изменения, соответствующие увеличению площади одной из зон. Все рисунки, иллюстрирующие зоны устойчивости, эволюцию зон устойчивости, спектры решений, графики движения маятников, фазовые портреты подготовлены с использованием системы математических расчётов Wolfram Mathematica.
\end{abstract}

Ключевые слова: обратный маятник, связанные осцилляторы, стабилизация, управление, зоны устойчивости.

Семенов Михаил Евгеньевич e-mail:mkl150@mail.ru

(i) Контент доступен под лицензией Creative Commons Attribution 4.0 License.

The content is available under Creative Commons Attribution 4.0 License. 


\section{ВВЕДЕНИЕ}

Теория колебаний нелинейных систем широко применяется при моделировании различных физических процессов и явлений[1], таких как колебания в электрических цепях, состоящих из нескольких взаимосвязанных контуров, молекул в жидкостях и твердых телах и т. д. В таких системах реализуются разнообразные дисперсионные зависимости, на основе которых исследуется распространение волн в нелинейных средах. Большинство таких моделей систем основывается на законах движения простейших связанных осцилляторов и их цепочек, динамика которых формализуется посредством линейных и нелинейных уравнений. Во множестве подобных задач рассматриваются колебания маятников с устойчивым нулевым положением [2-4]. Подробный обзор последних результатов в этой области приведён, например в [5].

\section{Анализ результатов предшествующих работ}

В то же время, в ряде практически важных задач (например, колебания поддерживающих контуров в строительстве, проблема стабилизации плазмы, стабилизация синтезированных биологических цепочек и т. п.) нулевое положение является неустойчивым. В связи с этим отметим классическую задачу стабилизации верхнего положения обратного маятника [6]. При решении этой задачи основное внимание уделялось проблеме стабилизации неустойчивого положения равновесия маятника путем движений нижней точки крепления. Этой проблеме посвящено огромное количество публикаций, достаточно подробный обзор которых содержится в [7]. Отметим, что стабилизация верхнего положения может быть достигнута различными способами: периодическими вертикальными осцилляциями нижней точки крепления, с использованием принципа обратной связи и др. Задача стабилизации маятника с помощью вертикальных осцилляций точки крепления хорошо изучена. Теоретическое объяснение этого явления было сделано Стефенсоном в 1908 году [8]. Физическое же объяснение ди- намической стабилизации перевернутого маятника вертикальными осцилляциями точки крепления было предложено академиком П. Л. Капицей в 1951 году, который также дал и оценку снизу частоты вибрации, при которой верхнее положение становится устойчивым [9].

Согласно $[9,10]$, уравнение динамики маятника единичной массы имеет вид:

$$
\ddot{\varphi}-\frac{1}{l}(g+\ddot{f}(t)) \sin \varphi=0,
$$

где $\varphi-$ угол отклонения маятника от вертикали, $l$ - длина маятника, $g$ - ускорение свободного падения, $f(t)-$ закон движения крепления.

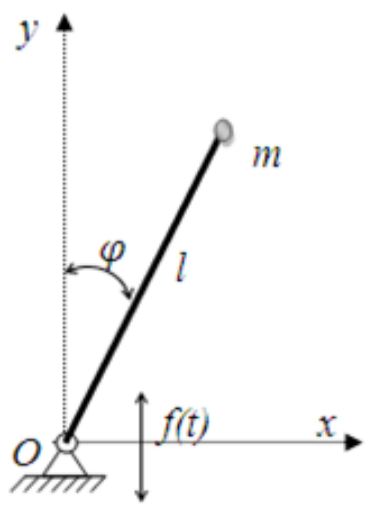

Рис. 1. Модель вертикального маятника с осииллирующим креплением

[Fig. 1. A model of a vertical pendulum with an oscillating pivot point]

При движении нижней точки крепления по гармоническому закону уравнение (1) сводится к хорошо известному уравнению Матьё [4]. Для адекватного описания динамики реальных физических и механических систем необходимо учитывать эффекты гистерезисной природы - люфты, упоры $[11,12]$. Исследование устойчивости таких систем детально проведены в [13-18].

Отметим, что проблеме стабилизации систем связанных неустойчивых осцилляторов посвящено совсем немного работ. Исследование возможности стабилизации таких систем при помощи горизонтальных движений проведено в [19-21]. Также представляет интерес другая задача - возможность управления различными конфигурациями цепочек с помощью вертикальных осцилляций точки крепления. 
В настоящей работе исследуется система, состоящая из двух перевернутых маятников, соединённых пружиной, с периодическим управляющим воздействием на один из них.

\section{Методы и материалы исследования (теоретическая часть)}

Ниже рассматривается система, в которой основание одного из маятников находится на подвижной планке, которая может перемещаться вдоль вертикальной оси, основание второго статически зафиксировано. Физическая модель изучаемой системы приведена на рис. 2.

При этом отметим, что при жёсткости пружины $k=0$, получается система из двух одинаковых маятников с управлением одним из них. В таком случае движение маятника на планке описывается уравнением Матьё, второй маятник описывается обычным уравнением гармонических колебаний. В случае $k \rightarrow \infty$, связь становится жёсткой (пружину можно заменить нерастяжимым стержнем) и маятники движутся как один. Таким образом, в обоих случаях для стабилизации системы достаточно воспользоваться известными результатами $[9,10]$.

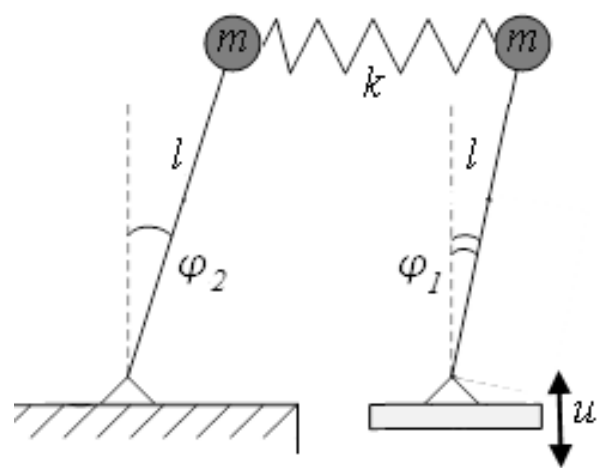

Рис. 2. Физическая модель связанных маятников

[Fig. 2. The physical model of coupled pendulums]

Ниже рассматривается случай, когда жёсткость пружины $k \in(0 ; \infty)$. Будем считать, что планка осуществляет движение так, что её ускорение изменяется периодически с частотой $\omega$, а амплитуда колебаний равна $A \omega^{2}$. Это соответствует тому, что линеаризованное уравнение движения описанной выше системы будет иметь вид:

$$
\left\{\begin{array}{c}
\ddot{\varphi}_{1}=\frac{1}{l}\left(g+A \omega^{2} w(t)\right) \varphi_{1}+\frac{k l^{2}}{J}\left(\varphi_{2}-\varphi_{1}\right) \\
\ddot{\varphi}_{2}=\frac{m g l}{J} \varphi_{2}-\frac{k l^{2}}{J}\left(\varphi_{2}-\varphi_{1}\right) \\
w(t)=-\operatorname{sign}(\sin (\omega t)) \\
\varphi_{1}(0)=\varphi_{11} \quad \dot{\varphi}_{1}(0)=\varphi_{12} \\
\varphi_{2}(0)=\varphi_{21} \quad \dot{\varphi}_{2}(0)=\varphi_{22}
\end{array}\right.
$$

где $w(t)-$ ускорение основания маятников, $J$ - момент инерции маятника, $k$ - жёсткость пружины.

Таким образом, задача заключается в следующем:

1) Выбор такого режима управления, который бы обеспечивал стабилизацию системы.

2) Определение зон устойчивости в пространстве параметров (амплитуда-частота), обеспечивающих стабилизацию.

Перейдем к безразмерным единицам, сделав в (2) следующую замену:

$$
\tau=\omega t, \alpha=\frac{g}{l}, \beta=\frac{A \omega^{2}}{l}, \gamma=\frac{k l^{2}}{J} .
$$

В итоге имеем следующую систему:

$$
\begin{gathered}
\left\{\begin{array}{c}
\ddot{\varphi}_{1}=(\alpha+\beta \operatorname{sign}(\sin \tau)) \varphi_{1}+\gamma\left(\varphi_{2}-\varphi_{1}\right) \\
\ddot{\varphi}_{2}=\alpha \varphi_{2}-\gamma\left(\varphi_{2}-\varphi_{1}\right)=0
\end{array}\right. \\
\varphi_{1}(0)=\varphi_{11} \quad \dot{\varphi}_{1}(0)=\varphi_{12} \\
\varphi_{2}(0)=\varphi_{21} \quad \dot{\varphi}_{2}(0)=\varphi_{22}
\end{gathered}
$$

Перепишем (4) в виде системы дифференциальных уравнений первого порядка

$$
\begin{aligned}
& \left\{\begin{array}{l}
\dot{z}_{1}=z_{2} \\
\dot{z}_{2}=p(\tau) z_{1}+\gamma z_{3} \\
\dot{z}_{3}=z_{4} \\
\dot{z}_{4}=(\alpha-\gamma) z_{3}+\gamma z_{1}
\end{array}\right. \\
& \varphi_{1}(0)=\varphi_{11} \quad \dot{\varphi}_{1}(0)=\varphi_{12} \\
& \varphi_{2}(0)=\varphi_{21} \quad \dot{\varphi}_{2}(0)=\varphi_{22}
\end{aligned}
$$

где $p(\tau)=\alpha+\beta \operatorname{sign}(\sin \tau)-\gamma$. Матрица полученной системы имеет следующий вид:

$$
\mathbf{P}(\tau)=\left(\begin{array}{cccc}
0 & 1 & 0 & 0 \\
p(\tau) & 0 & \gamma & 0 \\
0 & 0 & 0 & 1 \\
\gamma & 0 & (\alpha-\gamma) & 0
\end{array}\right) .
$$


В силу сделанных предположений матрица $\mathbf{P}(\tau)$, является периодической функцией времени с периодом $2 \pi$, так что для любого момента времени $\tau$ справедливо равенство $\mathbf{P}(\tau+2 \pi) \equiv \mathbf{P}(\tau)$.

Будем говорить, что уравнение (4) устойчиво или неустойчиво по Лагранжу, если устойчива или, соответственно, неустойчива система (5). То есть все решения $z(\tau)$ ограничены на $[0, \infty)$. В силу периодичности матрицы системы из результатов Флоке [13] следует, что задача исследования устойчивости сводится к нахождению фундаментальной матрицы решений в момент $2 \pi-$ матрицы монодромии и оценке ее собственных значений (мультипликаторов). Для устойчивости системы необходимо и достаточно, чтобы значения всех мультипликаторов находились внутри единичного круга:

$$
|\lambda|<1
$$

В силу того, что матрица $\mathbf{P}(\tau)-$ кусочно-постоянная, то фундаментальная система решений и, следовательно, матрица монодромии могут быть представлены в явном виде. Для этого рассмотрим поведение кусочно-постоянной функции $r(\tau)=\operatorname{sign}(\sin \tau)$ с периодом $2 \pi$ и соответственно $p(\tau)$ (рис. 3 ).

Из рис. 3 видно, что система (4) на промежутке $(0,2 \pi)$ может быть описана двумя линейными системами с постоянными коэффициентами:

$$
\left\{\begin{array}{l}
\dot{z}_{1}^{1}=z_{2}^{1} \\
\dot{z}_{2}^{1}=(\alpha+\beta-\gamma) z_{1}^{1}+\gamma z_{3}^{1} \\
\dot{z}_{3}^{1}=z_{4}^{1} \\
\dot{z}_{4}^{1}=(\alpha-\gamma) z_{3}^{1}+\gamma z_{1}^{1}
\end{array} \quad \tau \in[0, \pi]\right.
$$

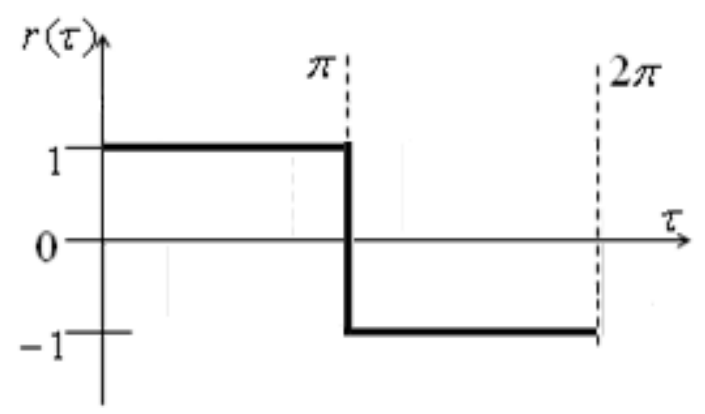

$$
\left\{\begin{array}{l}
\dot{z}_{1}^{1}=z_{2}^{1} \\
\dot{z}_{2}^{1}=(\alpha-\beta-\gamma) z_{1}^{1}+\gamma z_{3}^{1} \quad \tau \in[\pi, 2 \pi] \\
\dot{z}_{3}^{1}=z_{4}^{1} \\
\dot{z}_{4}^{1}=(\alpha-\gamma) z_{3}^{1}+\gamma z_{1}^{1}
\end{array}\right.
$$

Так как фундаментальная матрица должна быть непрерывна, то решения систем (7)-(8) должны совпадать в момент времени $\pi$ :

$$
\mathbf{Z}^{1}(0)=\mathbf{E}, \quad \mathbf{Z}^{1}(\pi)=\mathbf{Z}^{2}(\pi),
$$

где $\mathbf{E}$ - единичная матрица. Последовательно интегрируя системы (7)-(8) получим:

$$
\mathbf{Z}^{1}(\tau)=\left(\begin{array}{llll}
z_{1} & z_{6} & z_{5} & z_{3} \\
z_{7} & z_{1} & z_{4} & z_{5} \\
z_{5} & z_{3} & z_{2} & z_{8} \\
z_{4} & z_{5} & z_{9} & z_{2}
\end{array}\right),
$$

где $z_{1}=\operatorname{ch}\left(\tau a_{1}\right) a_{3}+\operatorname{ch}\left(\tau a_{2}\right) a_{4}$,

$$
z_{2}=\operatorname{ch}\left(\tau a_{1}\right) a_{4}+\operatorname{ch}\left(\tau a_{2}\right) a_{3} \text {, }
$$$$
z_{3}=\operatorname{sh}\left(\tau a_{1}\right) a_{9}-\operatorname{sh}\left(\tau a_{2}\right) a_{8} \text {, }
$$$$
z_{4}=\operatorname{sh}\left(\tau a_{1}\right) a_{10}+\operatorname{sh}\left(\tau a_{2}\right) a_{11} \text {, }
$$$$
z_{5}=\left(\operatorname{ch}\left(\tau a_{1}\right)-\operatorname{ch}\left(\tau a_{2}\right)\right) a_{5} \text {, }
$$$$
z_{6}=\operatorname{sh}\left(\tau a_{1}\right) a_{7}+\operatorname{sh}\left(\tau a_{2}\right) a_{6} \text {, }
$$$$
z_{7}=\operatorname{sh}\left(\tau a_{1}\right) a_{13}+\operatorname{sh}\left(\tau a_{2}\right) a_{12} \text {, }
$$$$
z_{8}=\operatorname{sh}\left(\tau a_{1}\right) a_{15}+\operatorname{sh}\left(\tau a_{2}\right) a_{14} \text {, }
$$$$
z_{9}=\operatorname{sh}\left(\tau a_{1}\right) a_{17}+\operatorname{sh}\left(\tau a_{2}\right) a_{16} \text {. }
$$

$$
\mathbf{Z}^{2}(\tau)=\mathbf{Z}^{1}(\pi) \times\left(\begin{array}{cccc}
z_{1} & z_{6} & z_{5} & z_{3} \\
z_{7} & z_{1} & z_{4} & z_{5} \\
z_{5} & z_{3} & z_{2} & z_{8} \\
z_{4} & z_{5} & z_{9} & z_{2}
\end{array}\right),
$$

где $z_{1}=\operatorname{ch}\left((\tau-\pi) c_{1}\right) a_{3}+\operatorname{ch}\left((\tau-\pi) a_{1}\right) a_{4}$,

$$
z_{2}=\operatorname{ch}\left((\tau-\pi) a_{1}\right) a_{3}+\operatorname{ch}\left((\tau-\pi) c_{1}\right) a_{4},
$$

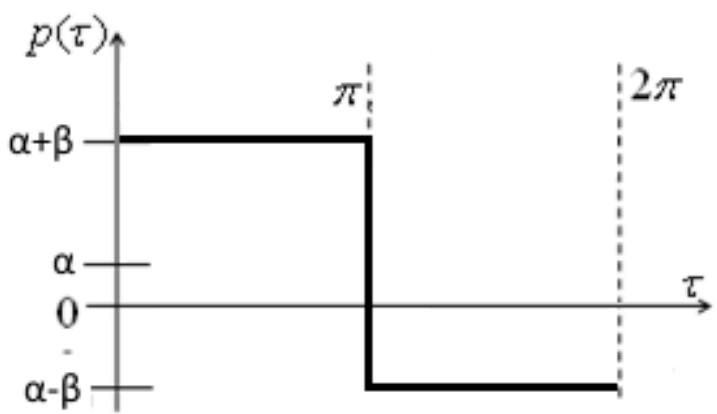

Рис. 3. Графики функций $r(\tau)$ и $p(\tau)$

[Fig. 3. Graphs of the functions $r(\tau)$ and $p(\tau)$ ] 
Стабилизация системы связанных обратных маятников вертикальным управлением

$$
\begin{aligned}
& z_{3}=\operatorname{sh}\left((\tau-\pi) a_{1}\right) c_{4}-\operatorname{sh}\left((\tau-\pi) c_{1}\right) a_{8}, \\
& z_{4}=\operatorname{sh}\left((\tau-\pi) a_{1}\right) c_{2}+\operatorname{sh}\left((\tau-\pi) c_{1}\right) c_{3}, \\
& z_{5}=\left(\operatorname{ch}\left((\tau-\pi) a_{1}\right)-\operatorname{ch}\left((\tau-\pi) c_{1}\right)\right) a_{5}, \\
& z_{6}=\operatorname{sh}\left((\tau-\pi) c_{1}\right) a_{14}+\operatorname{sh}\left((\tau-\pi) a_{1}\right) a_{15}, \\
& z_{7}=\operatorname{sh}\left((\tau-\pi) a_{1}\right) c_{5}+\operatorname{sh}\left((\tau-\pi) c_{1}\right) c_{6}, \\
& z_{8}=\operatorname{sh}\left((\tau-\pi) c_{1}\right) a_{6}+\operatorname{sh}\left((\tau-\pi) a_{1}\right) a_{7}, \\
& z_{9}=\operatorname{sh}\left((\tau-\pi) a_{1}\right) c_{7}+\operatorname{sh}\left((\tau-\pi) c_{1}\right) c_{8} .
\end{aligned}
$$

Тогда, полагая в $\mathbf{Z}^{2}(\tau) \quad \tau=2 \pi$, получим матрицу монодромии системы (4) в следующем виде:

$$
=\left(\begin{array}{llll}
h_{1} & h_{6} & h_{5} & h_{3} \\
h_{7} & h_{1} & h_{4} & h_{5} \\
h_{5} & h_{3} & h_{2} & h_{8} \\
h_{4} & h_{5} & h_{9} & h_{2}
\end{array}\right) \times\left(\begin{array}{llll}
h_{11} & h_{16} & h_{15} & h_{13} \\
h_{17} & h_{11} & h_{14} & h_{15} \\
h_{15} & h_{13} & h_{12} & h_{18} \\
h_{14} & h_{15} & h_{19} & h_{12}
\end{array}\right),
$$

где $h_{1}=\operatorname{ch}\left(\pi a_{1}\right) a_{3}+\operatorname{ch}\left(\pi a_{2}\right) a_{4}$,

$$
\begin{aligned}
& h_{2}=\operatorname{ch}\left(\pi a_{2}\right) a_{3}+\operatorname{ch}\left(\pi a_{1}\right) a_{4}, \\
& h_{3}=\operatorname{sh}\left(\pi a_{1}\right) a_{9}-\operatorname{sh}\left(\pi a_{2}\right) a_{8}, \\
& h_{4}=\operatorname{sh}\left(\pi a_{1}\right) a_{10}-\operatorname{sh}\left(\pi a_{2}\right) a_{11}, \\
& h_{5}=\left(\operatorname{ch}\left(\pi a_{1}\right)-\operatorname{ch}\left(\pi a_{2}\right)\right) a_{5}, \\
& h_{6}=\operatorname{sh}\left(\pi a_{1}\right) a_{7}+\operatorname{sh}\left(\pi a_{2}\right) a_{6}, \\
& h_{7}=\operatorname{sh}\left(\pi a_{1}\right) a_{13}+\operatorname{sh}\left(\pi a_{2}\right) a_{12}, \\
& h_{8}=\operatorname{sh}\left(\pi a_{1}\right) a_{15}+\operatorname{sh}\left(\pi a_{2}\right) a_{14}, \\
& h_{9}=\operatorname{sh}\left(\pi a_{1}\right) a_{17}+\operatorname{sh}\left(\pi a_{2}\right) a_{16}, \\
& h_{11}=\operatorname{ch}\left(\pi c_{1}\right) a_{3}+\operatorname{ch}\left(\pi a_{1}\right) a_{4}, \\
& h_{12}=\operatorname{ch}\left(\pi a_{1}\right) a_{3}+\operatorname{ch}\left(\pi c_{1}\right) a_{4}, \\
& h_{13}=\operatorname{sh}\left(\pi a_{1}\right) c_{4}-\operatorname{sh}\left(\pi c_{1}\right) a_{8}, \\
& h_{14}=\operatorname{sh}\left(\pi a_{1}\right) c_{2}+\operatorname{sh}\left(\pi c_{1}\right) c_{3}, \\
& h_{15}=\left(\operatorname{ch}\left(\pi a_{1}\right)-\operatorname{ch}\left(\pi c_{1}\right)\right) a_{5}, \\
& h_{16}=\operatorname{sh}\left(\pi a_{1}\right) a_{14}+\operatorname{sh}\left(\pi c_{1}\right) a_{15}, \\
& h_{17}=\operatorname{sh}\left(\pi a_{1}\right) c_{5}+\operatorname{sh}\left(\pi c_{1}\right) c_{6}, \\
& h_{18}=\operatorname{sh}\left(\pi c_{1}\right) a_{6}+\operatorname{sh}\left(\pi a_{1}\right) a_{7}, \\
& h_{19}=\operatorname{sh}\left(\pi a_{1}\right) c_{7}+\operatorname{sh}\left(\pi c_{1}\right) c_{8} .
\end{aligned}
$$

В качестве составляющих элементов матриц $\mathbf{Z}$ и $\mathbf{H}$ были использованы следующие обозначения: $e_{1}=\alpha-\gamma, e_{2}=\sqrt{2} \gamma$, $b_{1}=\sqrt{\beta^{2}+4 \gamma^{2}}, b_{2}=2 e_{1}+\beta, b_{3}=b_{1}+\beta$, $b_{4}=b_{1}-\beta, b_{5}=\frac{1}{b_{1} \sqrt{2\left(b_{1}+b_{2}\right)}}$,

$$
\begin{aligned}
& b_{6}=\frac{1}{b_{1} \sqrt{2\left(b_{2}-b_{1}\right)}}, b_{7}=\frac{1}{2 b_{1}}, a_{1}=\frac{b_{7}}{b_{5}}, a_{2}=\frac{b_{7}}{b_{6}}, \\
& a_{3}=b_{3} b_{7}, a_{4}=b_{4} b_{7}, a_{5}=2 \gamma b_{7}, a_{6}=b_{4} b_{6}, a_{7}=b_{3} b_{5}, \\
& a_{8}=2 \gamma b_{6}, a_{9}=2 \gamma b_{5}, a_{10}=a_{1} a_{5}, a_{11}=\frac{a_{8}\left(2 e_{1}-b_{4}\right)}{2}, \\
& a_{12}=\left(b_{4}\left(e_{1}+\beta\right)-e_{2}^{2}\right) b_{6}, \\
& a_{13}=\left(b_{3}\left(e_{1}+\beta\right)+e_{2}^{2}\right) b_{5}, a_{14}=b_{4} b_{5}, a_{15}=b_{3} b_{6}, \\
& a_{16}=\left(b_{3} e_{1}-e_{2}^{2}\right) b_{6}, a_{17}=\left(e_{2}^{2}-\alpha b_{3}-b_{4} \gamma\right) b_{5}, \\
& d_{1}=\alpha-4 \gamma, d_{2}=d_{1}-\beta, c_{1}=\sqrt{e_{1}-\frac{b_{3}}{2}}, \\
& c_{2}=2 e_{2} b_{5}\left(e_{1}+\sqrt{2} b_{4}\right), c_{3}=\sqrt{2} e_{2} b_{6}\left(2 b_{3}-e_{1}\right), \\
& c_{4}=2 e_{2} b_{5}, c_{5}=\left(b_{4} d_{2}+\sqrt{2} e_{2}^{2}\right) b_{5}, \\
& c_{6}=\left(b_{3} d_{2}-e_{2}^{2}\right) b_{6}, c_{7}=\left(b_{3} d_{1}+\sqrt{2} e_{2}^{2}\right) b_{5}, \\
& c_{8}=\left(b_{4} d_{1}-e_{2}^{2}\right) b_{6} .
\end{aligned}
$$

Характеристическое уравнение для матрицы $\mathbf{Z}(2 \pi)$ имеет вид:

$$
\begin{gathered}
=\left|\begin{array}{cccc}
h_{11}-\lambda & h_{12} & h_{13} & h_{14} \\
h_{21} & h_{22}-\lambda & h_{23} & h_{24} \\
h_{31} & h_{32} & h_{33}-\lambda & h_{34} \\
h_{41} & h_{42} & h_{43} & h_{44}-\lambda
\end{array}\right|= \\
=\lambda^{4}+q_{1} \lambda^{3}+q_{2} \lambda^{2}+q_{3} \lambda+q_{4}=0, \\
\text { где } q_{4}=(-1)^{4} e^{\int^{T} S p(\mathbf{P}(\tau)) d \tau}=1[14] .
\end{gathered}
$$

Для идентификации зон устойчивости в пространстве параметров можно воспользоваться критерием Рауса - Гурвица. Для этого необходимо сделать замену $\lambda=\frac{1+\mu}{1-\mu}$ (это отображение сопоставляет единичному кругу левую часть комплексной плоскости), получив уравнение с комплексными коэффициентами вида $u_{0} \mu^{4}+u_{1} \mu^{3}+u_{2} \mu^{2}+u_{3} \mu+u_{4}=0$. Для того, чтобы корни соответствующего уравнения лежали в левой части комплексной плоскости, а как следствие, корни уравнения (10) лежали внутри единичного круга достаточно, чтобы определители главных миноров матрицы Гурвица, составленной из коэффициентов $u_{0} \ldots u_{4}$ были положительными. Второй способ связан с прямым вычислением корней характеристического уравнения (10). 
Решения уравнения (10) имеют следующий вид:

$$
\begin{aligned}
& \lambda_{1}=-\frac{1}{2}\left(\frac{q_{1}}{2}-y_{6}+\sqrt{y_{8}+y_{7}}\right), \\
& \lambda_{2}=-\frac{1}{2}\left(\frac{q_{1}}{2}-y_{6}-\sqrt{y_{8}+y_{7}}\right), \\
& \lambda_{3}=-\frac{1}{2}\left(\frac{q_{1}}{2}+y_{6}+\sqrt{y_{8}-y_{7}}\right), \\
& \lambda_{4}=-\frac{1}{2}\left(\frac{q_{1}}{2}+y_{6}-\sqrt{y_{8}-y_{7}}\right),
\end{aligned}
$$

где $\quad y_{1}=12+q_{2}^{2}-3 q_{1} q_{3}, \quad y_{2}=27 q_{1}^{2}-72 q_{2}+$ $+2 q_{2}^{3}-9 q_{1} q_{2} q_{3}+27 q_{3}^{2}, \quad y_{3}=-q_{1}^{3}+4 q_{1} q_{2}-8 q_{3}$, $y_{4}=\frac{q_{1}^{2}}{4}-\frac{2 q_{2}}{3}, \quad y_{5}=\frac{\sqrt[3]{2} y_{1}}{3 \sqrt[3]{y_{2}+\sqrt{-4 y_{1}^{3}+y_{2}^{2}}}}+$ $+\frac{\sqrt[3]{y_{2}+\sqrt{-4 y_{1}^{3}+y_{2}^{2}}}}{\sqrt[3]{32}}, \quad y_{6}=\sqrt{y_{4}+y_{5}}, \quad y_{7}=\frac{y_{3}}{4 y_{6}}$, $y_{8}=2 y_{4}-y_{5}$.

Учитывая (6) и (11) для устойчивости системы необходимо выполнение неравенства $\max _{i}\left|\lambda_{i}\right|<1$. Имея ввиду функциональную зависимость параметров характеристического уравнения от амплитуды, частоты и жёсткости пружины, построим зоны устойчивости в пространстве параметров.

\section{Результаты исследований и их обсуждение}

Границами зоны устойчивости являются линии в пространстве параметров, соответствующие значениям максимального собственного числа, равного единице. Для построения этих областей последовательно зафиксируем значения жёсткости пружины на уровнях $k=20,35,50,70,85,100$. Результаты получены численно, с помощью пакета Wolfram Mathematica.

Из рис. 4 видно, что геометрия зон устойчивости претерпевает изменения, соответствующие увеличению площади второй зоны. Заметим, также, что полученные результаты схожи с графиками, полученными в $[16,22,23]$.

Чтобы проиллюстрировать эволюцию изменения зон устойчивости в зависимости от жёсткости пружины, приведём сдвиг левой границы правой зоны устойчивости.
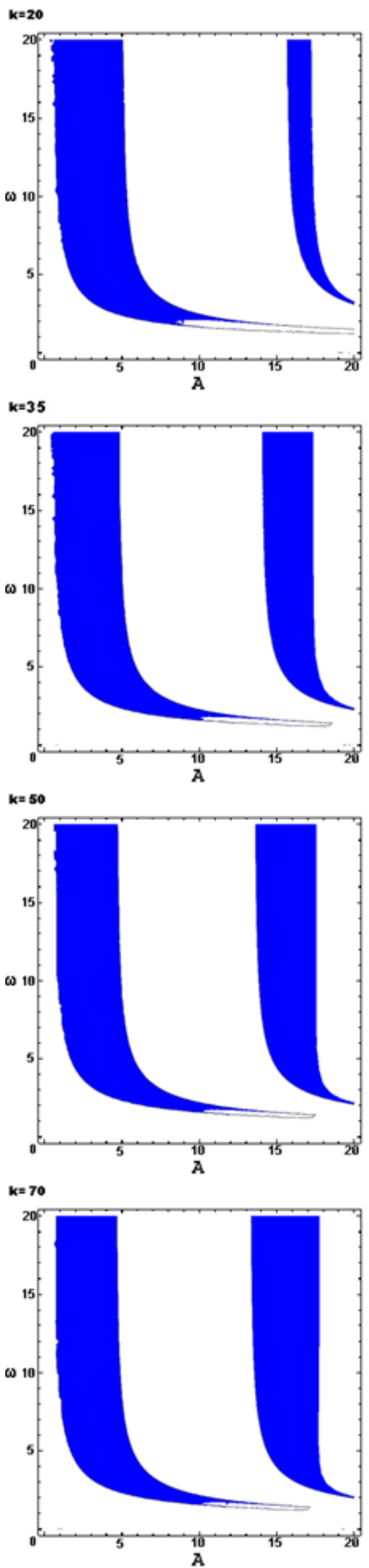

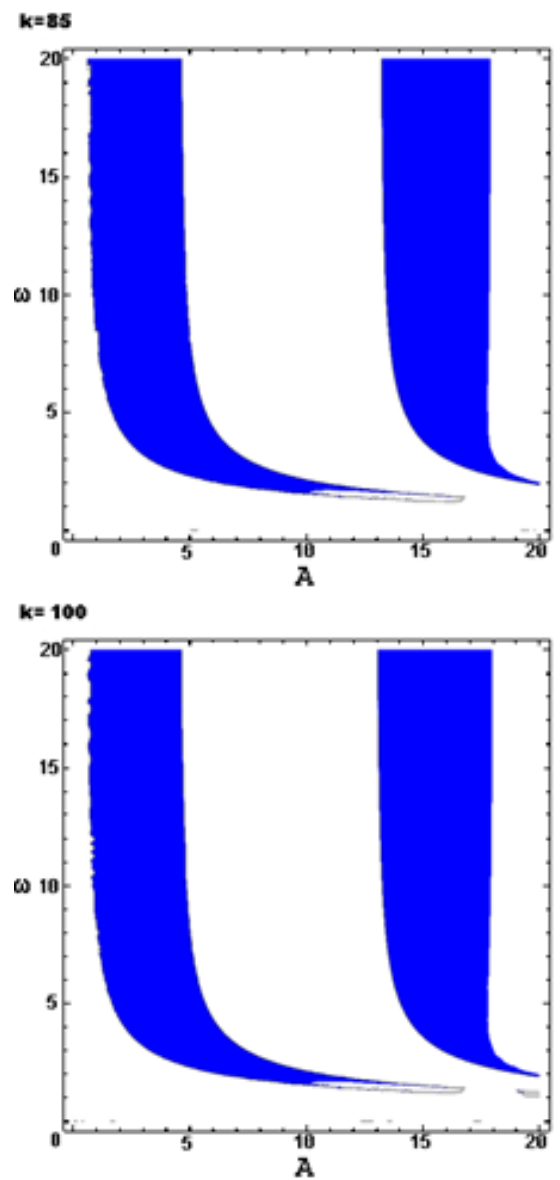

Рис. 4. Графики областей устойчивости при различных жёсткостях пружины $k$

[Fig. 4. Graphs of stability areas with different

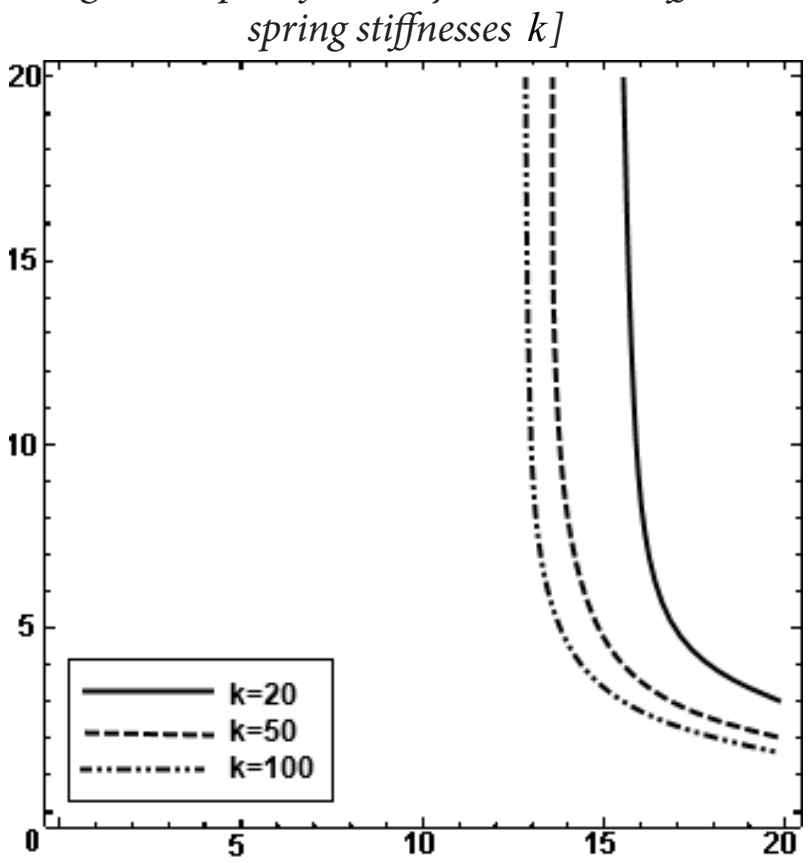

Рис. 5. Сдвиг левой границь правой области устойчивости при различных $k$

[Fig. 5. A shift of the right stability region's left boundary for different $k$ values]
Портреты и зависимости фазовых координат (размерных) от времени для значений параметров системы: $l=1 \mathrm{M}, k=95 \mathrm{H} / \mathrm{M}$, $A=10 \mathrm{M}, \omega=20$ Гц, $\varphi_{1}(0)=0.01, \dot{\varphi}_{1}(0)=-0.01$, $\varphi_{2}(0)=-0.03, \dot{\varphi}_{2}(0)=0.02$. (рис. 6).

В заключении отметим, что полученные движения соответствуют почти периодическим функциям: спектры полученных решений имеют две ярко выраженные и две побочные гармоники (рис. 7).

\section{Периодические режимы}

Рассмотрим поведение движения маятника при параметрах, лежащих на границах области устойчивости, что возвращаясь к характеристическому уравнению матрицы монодромии (9) относится к трём случаям: когда $q_{1}=-4, q_{1}=0, q_{1}=4$. Тогда мультипликаторы будут принимать следующие значения: $\lambda_{1}=\lambda_{2}=\lambda_{3}=\lambda_{4}=1, \lambda_{1}=\lambda_{2}=-1 ; \quad \lambda_{3}=\lambda_{4}=1$ и $\lambda_{1}=\lambda_{2}=\lambda_{3}=\lambda_{4}=-1$ соответственно.

Если $\lambda_{1}=\lambda_{2}=\lambda_{3}=\lambda_{4}=1$, то соответствующее нормальное решение будет удовлетворять равенству $\mathbf{Z}(t+2 \pi)=\mathbf{Z}(t)$, следовательно, уравнение (2) имеет периодическое решение, период которого совпадает с периодом его коэффициентов $T_{1}=\frac{2 \pi}{\omega}$.

Если $\quad \lambda_{1}=\lambda_{2}=-1 ; \quad \lambda_{3}=\lambda_{4}=1 \quad$ или $\lambda_{1}=\lambda_{2}=\lambda_{3}=\lambda_{4}=-1, \quad$ то соответствующее нормальное решение будет удовлетворять равенству $\mathbf{Z}(t+2 \pi)=-\mathbf{Z}(t)$, а еще через один период $\mathbf{Z}(t+4 \pi)=-\mathbf{Z}(t+\pi)=\mathbf{Z}(t)$, следовательно, уравнение (2) имеет периодические решения, период которых $T_{2,3}=\frac{4 \pi}{\omega}[25]$.

Таким образом, периодические режимы будут иметь место, если будет выполнено одно из соотношений:

$$
\begin{gathered}
q_{1}=4, \text { для периода колебаний } T_{1} \\
q_{1}=-4 \text { или } q_{1}=0, \\
\text { для периода колебаний } T_{2}
\end{gathered}
$$

Это необходимые, но не достаточные условия, поскольку не для любых начальных значений при заданном управлении с параметрами, удовлетворяющими одному из указанных равенств, будут существовать периодические решения. 

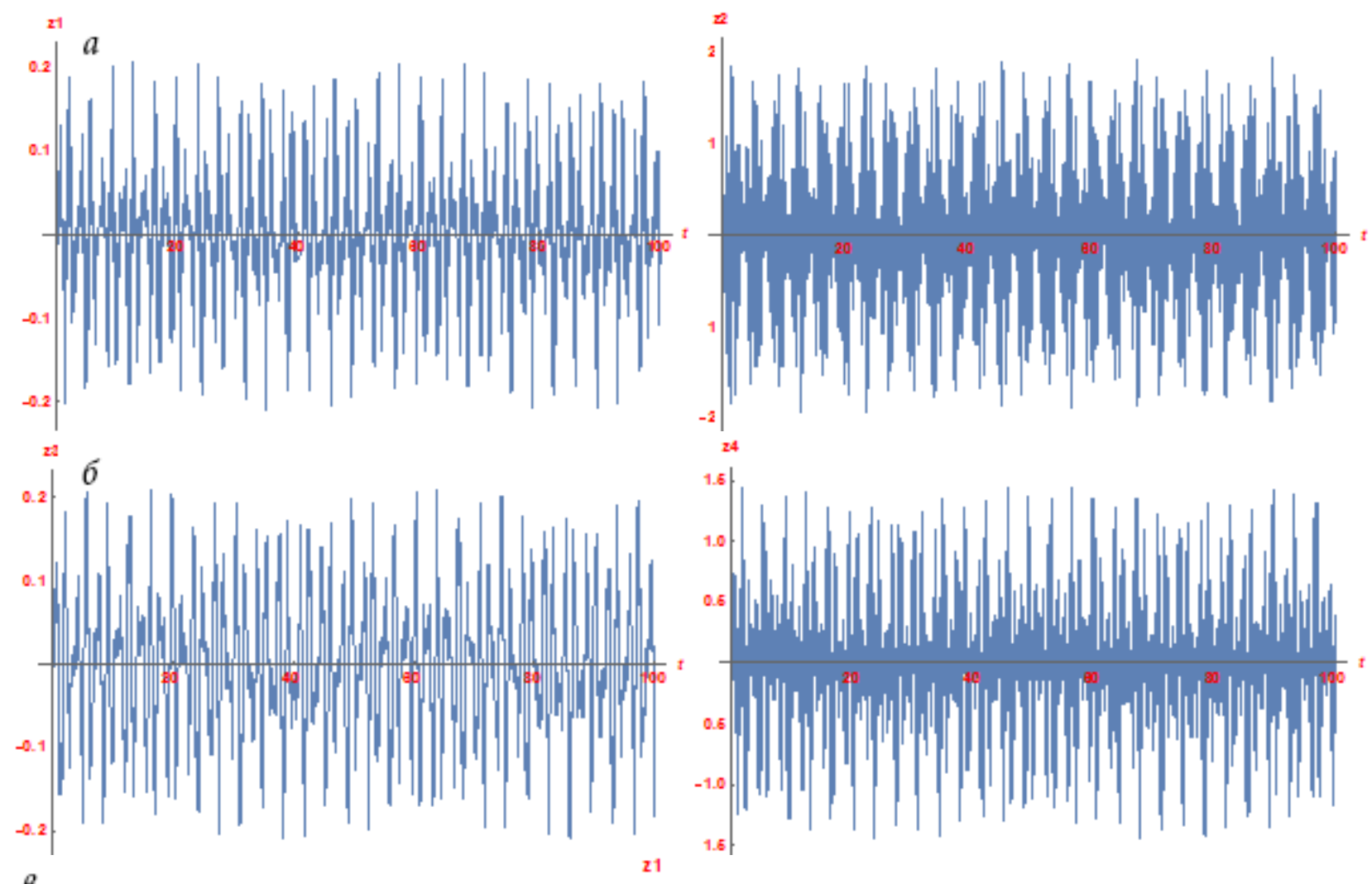

B
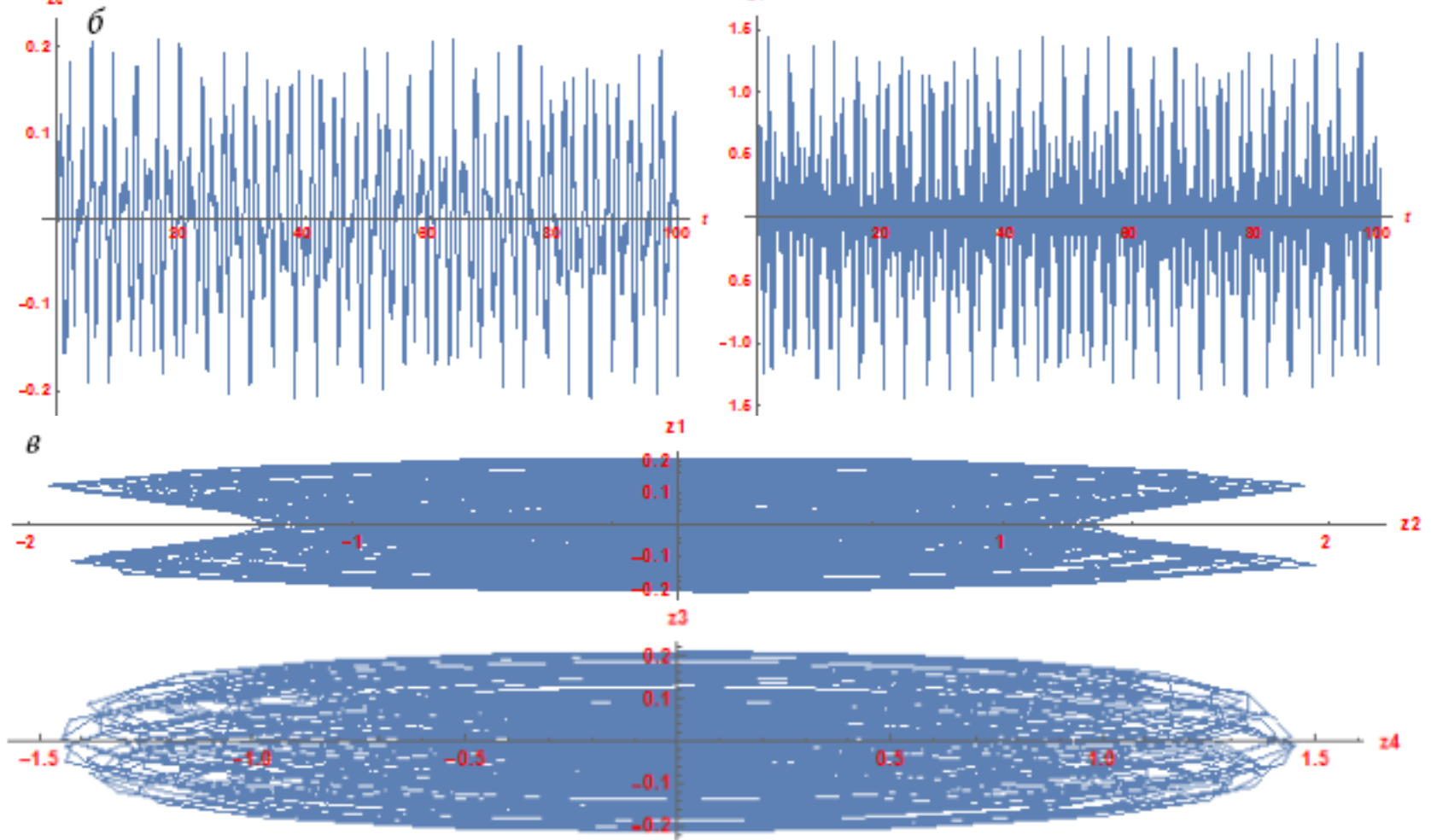

Рис. б. а) Графики характеристик движения первого маятника б) Графики характеристик движения второго маятника в) Фазовые плоскости

[Fig. 6. a) Graphs of the motion characteristics for the first pendulum. b) Graphs of the motion characteristics for the second pendulum.c) Phase planes]

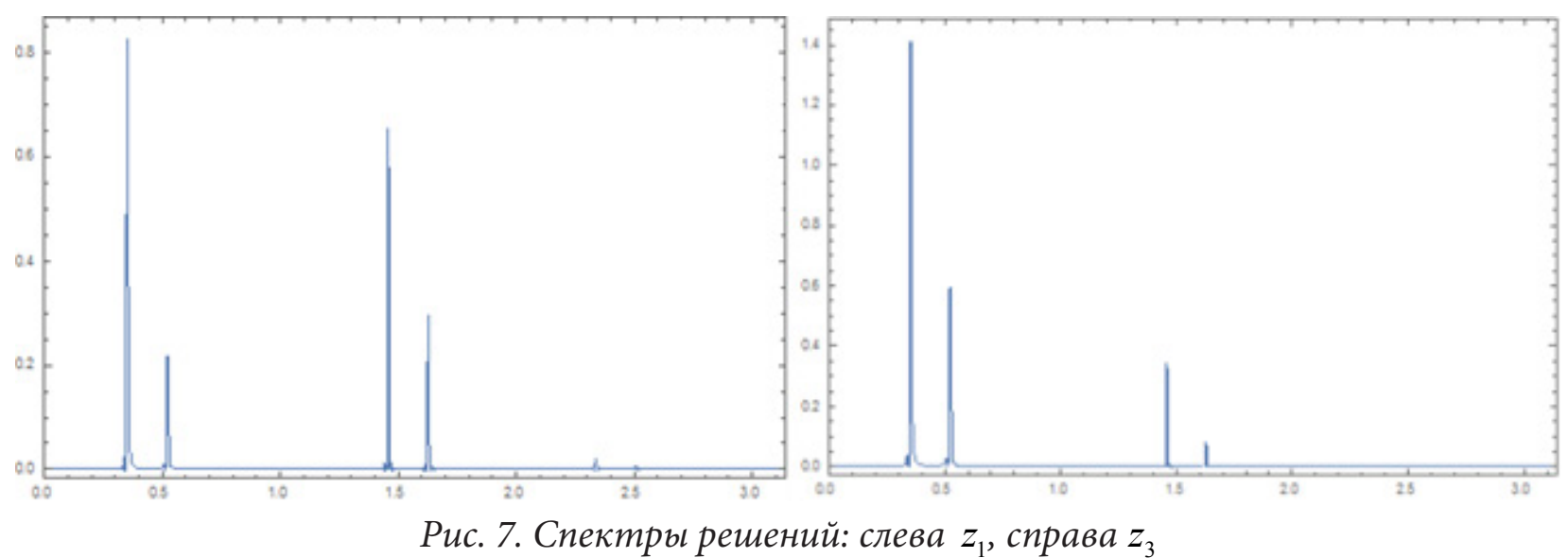

[Fig. 7. Spectra of solutions: $z_{1}$ (left) and $z_{3}$ (right)] 
Отметим, что для рассматриваемого здесь класса управлений, начальные условия лежат в I и III четвертях.

Определим следующее начальное условие $\left(\varphi_{10}, \varphi_{20}, \varphi_{30}, \varphi_{40}\right)$, и рассмотрим периодические колебания с периодом $T_{1}$, тогда должно быть справедливо равенство $\mathbf{Z}(0+T)=$ $=\mathbf{H Z}(0)=\mathbf{Z}(0)$, то есть соответствующий вектор является собственным вектором матрицы монодромии, отвечающим единичному собственному значению:

$$
\left(\begin{array}{llll}
h_{11} & h_{12} & h_{13} & h_{14} \\
h_{21} & h_{22} & h_{23} & h_{24} \\
h_{31} & h_{32} & h_{33} & h_{34} \\
h_{41} & h_{42} & h_{43} & h_{44}
\end{array}\right)\left(\begin{array}{l}
\varphi_{10} \\
\varphi_{20} \\
\varphi_{30} \\
\varphi_{40}
\end{array}\right)=\left(\begin{array}{c}
\varphi_{10} \\
\varphi_{20} \\
\varphi_{30} \\
\varphi_{40}
\end{array}\right) .
$$

Следовательно, начальные условия должны удовлетворять условиям:

$$
\begin{aligned}
& \varphi_{10}=\frac{h_{12}}{1-h_{11}} \varphi_{20}+\frac{h_{13}}{1-h_{11}} \varphi_{30}+\frac{h_{14}}{1-h_{11}} \varphi_{40}, \\
& \varphi_{20}=\frac{h_{21}}{1-h_{22}} \varphi_{10}+\frac{h_{23}}{1-h_{22}} \varphi_{30}+\frac{h_{24}}{1-h_{22}} \varphi_{40}, \\
& \varphi_{30}=\frac{h_{31}}{1-h_{33}} \varphi_{10}+\frac{h_{32}}{1-h_{33}} \varphi_{20}+\frac{h_{42}}{1-h_{33}} \varphi_{40}, \\
& \varphi_{40}=\frac{h_{41}}{1-h_{44}} \varphi_{10}+\frac{h_{42}}{1-h_{44}} \varphi_{20}+\frac{h_{43}}{1-h_{44}} \varphi_{30} .
\end{aligned}
$$

Введём новые параметры посредством следующих обозначений: $\frac{h_{12}}{1-h_{11}}=n_{12}, \frac{h_{13}}{1-h_{11}}=n_{13}$, $\ldots, \frac{h_{43}}{1-h_{44}}=n_{43}$, тогда:

$$
\begin{gathered}
\varphi_{20}=\frac{n_{21}+n_{24} n_{41}}{1-n_{24} n_{42}} \varphi_{10}+\frac{n_{23}+n_{24} n_{43}}{1-n_{24} n_{42}} \varphi_{30}, \\
\varphi_{40}=\frac{n_{41}+n_{42} n_{21}}{1-n_{42} n_{24}} \varphi_{10}+\frac{n_{43}+n_{42} n_{23}}{1-n_{42} n_{24}} \varphi_{30},
\end{gathered}
$$

т. е. начальные условия, отвечающие периодическим решениям лежат на плоскости

$$
\begin{aligned}
& \dot{\varphi}_{20}=K_{11}^{1} \varphi_{10}+K_{12}^{1} \varphi_{30}, \\
& \dot{\varphi}_{40}=K_{21}^{1} \varphi_{10}+K_{22}^{1} \varphi_{30},
\end{aligned}
$$

где коэффициенты имеют следующие значения:

$$
\begin{aligned}
& K_{11}^{1}=\frac{h_{21}\left(1-h_{44}\right)+h_{24} h_{41}}{\left(1-h_{22}\right)\left(1-h_{44}\right)-h_{24} h_{42}}, \\
& K_{12}^{1}=\frac{h_{23}\left(1-h_{44}\right)+h_{24} h_{43}}{\left(1-h_{22}\right)\left(1-h_{44}\right)-h_{24} h_{42}}
\end{aligned}
$$

$$
\begin{aligned}
& K_{21}^{1}=\frac{h_{41}\left(1-h_{22}\right)+h_{42} h_{21}}{\left(1-h_{44}\right)\left(1-h_{22}\right)-h_{42} h_{24}}, \\
& K_{22}^{1}=\frac{h_{43}\left(1-h_{22}\right)+h_{42} h_{23}}{\left(1-h_{44}\right)\left(1-h_{22}\right)-h_{42} h_{24}} .
\end{aligned}
$$

Если для начальных условий $\left(\varphi_{10}, \varphi_{20}, \varphi_{30}, \varphi_{40}\right)$ можно найти пару параметров $A$ и $\omega$, лежащих на границе области устойчивости, удовлетворяющую равенству (17), то эта пара единственна. Обратное так же верно.

Аналогично определяется плоскость, соответствующая начальным условиям, отвечающим периодическим решениям с периодом $T_{2}$.

\section{ЗАКЛЮЧЕНИЕ}

В работе рассматривалась динамика системы, состоящей из неустойчивых связанных маятников, находящихся под воздействием сил инерции, обусловленных вертикальными осцилляциями нижней точки крепления одного из них. Было показано, что наличие жёсткой упругой связи между маятниками коренным образом меняет динамику системы. Также построены зоны устойчивости в пространстве естественных параметров, а также исследована эволюция зон устойчивости в зависимости от жёсткости пружины. Установлено наличие периодических режимов на границах зон устойчивости, доказана их неустойчивость.

\section{КОНФЛИКТ ИНТЕРЕСОВ}

Авторы декларируют отсутствие явных и потенциальных конфликтов интересов, связанных с публикацией настоящей статьи.

Работа выполнена при поддержке РФФИ, грант № 19-08-00158

\section{СПИСОК ЛИТЕРАТУРЫ}

1. Андронов А. А. Bитm А. А. Хайкин С. Э. Теория колебаний. М. : Наука, 1981. 568 с.

2. Баутин Н. Н. О числе предельных циклов, появляющихся при изменении коэффициентов из состояния равновесия типа фоку- 
са или центра, Математический сборник.1952, 30(72), 181-196.

3. Трубеиков Д. И., Рожнев А. Г. Линейные колебания и волны. М. : Издательство Физико-математической литературы, 2001. 416 с.

4. Магнус К. Колебания: Введение в исследование колебательных систем. Пер. с нем. М. : Мир, 1982. 304 с.

5. Осипов Г. В. Синхронизация в неоднородных сетях осцилляторов. Нижний Новгород, 2014. $135 \mathrm{c}$.

6. Колмогоров А. Н. О сохранении условно периодических движений при малом изменении функции Гамильтона. Доклады АН СССР, 1954. 98(4). 527-530.

7. Бутиков Е. И. Стабилизация перевернутого маятника (60 лет маятнику Капицы). Компьютерные инструменты в образовании, 2010. 5. 39-51.

8. Stephenson A. «On an induced stability», Phil. Mag, 1908. 15(233)

9. Капиนа П. Л. Маятник с вибрирующим подвесом. УФН, 1951. 44. 7-20.

10. Капица П. Л. Динамическая устойчивость маятника при колеблющейся точке подвеса. ЖЭТФ, 1951. 21. 588-597.

11. Нелепин Р. А. Методы исследования нелинейных систем автоматического управления. М. : Наука, 1979. 447 с.

12. Красносельский М. А., Покровский А. В. Системы с гистерезисом. М. : Наука, 1983. $271 \mathrm{c}$.

13. Плисс В. А. Нелокальные проблемы теории колебаний. М. : Наука, 1964. 367 с.

14. Меркин Д. Р. Введение в теорию устойчивости движения. М. : Наука, 1987. 304 с.

15. Матвеев М. Г., Семёнов М. Е., Шевлякова Д. В., Канищева О. И. Зоны устойчивости и периодические решения перевернутого маятника с гистерезисным управлением. Мехатроника. Автоматизация. Управление, 2012. 11. 8-14.

16. Семенов М. Е., Хатиф 3., Решетова О. О., Демчук А. А., Мелешенко П. А. Модель динамики обратного маятника с гистерезис- ным управлением. Вестник Воронеж. гос. унта. Сер. Физика. Математика, 2016. 4. 165-177.

17. Семёнов М. Е., Матвеев М. Г., Лебедев Г. Н., Соловъёв А. М. Стабилизация обратного гибкого маятника с гистерезисными свойствами. Мехатроника. Автоматизация.Управление. 2017. 8. 516-525. DOI: 10.17587/mau.18. 516-525.

18. Рянских А. В., Семенов М. Е., Рукавииын А. Г., Канищева О. И., Демчук А. А., Мелешенко П. А. Стабилизация обратного маятника на двухколесном транспортном средстве. Вестн. Южно-Ур. ун-та. Сер. Матем. Мех. Физ., 2017. Т. 9, вып. 3. 41-50. DOI: 10.14529/ mmph170306

19. Семенов М. Е., Соловъев А. М., Попов М. А. Стабилизация неустойчивых объектов: связанные осцилляторы. Труды МАИ, 2017. 93.

20. Solovyov A. M., Semenov M. E., Meleshenko P. A., Reshetova O. O., Popov M. A., Kabulova $E$. G. Hysteretic nonlinearity and unbounded solutions in oscillating systems. Procedia Engineering, 2017. 201. 549-555. DOI: 10.1016/j.proeng.2017.09.634

21. Semenov M. E., Solovev A. M., Popov M. A., Meleshenko P. A. Coupled inverted pendulums: stabilization problem. Archive Of Applied Mechanics. 2018. 88. 517-524.

22. Семёнов М. Е., Попов М. А., Канищева О. И. Управление системой нелинейно связанных перевернутых маятников, Журнал Сибирского Федерального Университета. Серия: Техника и Технологии. 2018. 11(3), 280290. DOI: $10.17516 / 1999-494 X-0040$

23. Неймарк Ю. И., Коган Н. Я., Савельев В. П. Динамические модели теории управления. М. : Наука, 1985. 400 с.

24. Красносельский M. А. Нелинейные почти периодические колебания. М. : Наука, 1970, 304 c.

25. Мирошник И. В. Теория автоматического управления. Нелинейные и оптимальные системы. СПб. : Питер, 2006. 272 с. 
Семенов Михаил Евгеньевич - д. ф.-м. н., профессор, ведущий научный сотрудник Федерального исследовательского центра «Единая геофизическая служба Российской академии наук»; профессор кафедры цифровых технологий, Воронежский Государственный Университет. E-mail: mkl150@mail.ru ORCID iD: https://orcid.org/0000-0002-3361-1102

Канищева Олеся Ивановна - к. ф.-м. н., доцент кафедры математики Военного учебно-научного центра Военно-воздушных сил «Военно-воздушная академия имени профессора Н. Е. Жуковского и Ю. А. Гагарина».

E-mail: oleka_olesya@mail.ru

ORCID iD: https://orcid.org/0000-0002-1830-4091

Попов Михаил Александрович - аспирант кафедры прикладной математики и механики, Воронежский государственный технический университет.

E-mail: soeltic@gmail.com

ORCID iD: https://orcid.org/0000-0003-4336-8190

DOI: https://doi.org/10.17308/sait.2020.1/2587

Received 26.08.2019

Accepted 15.03.2020

ISSN 1995-5499

\title{
STABILIZATION OF A COUPLED INVERTED PENDULUMS SYSTEM VIA VERTICAL FORCES
}

\author{
(C) 2020 M. E. Semenov ${ }^{\bowtie 1,2,3}$, O. I. Kanishcheva ${ }^{4}$, M. A. Popov ${ }^{3}$ \\ ${ }^{1}$ Geophysical Survey of Russia Academy of Sciences \\ 189, Lenin Ave., 249035 Obninsk, Kaluga Region, Russian Federation \\ ${ }^{2}$ Voronezh State University \\ 1, University Square, 394018 Voronezh, Russian Federation \\ ${ }^{3}$ Voronezh State Technical University \\ 84, 20 let Oktyabrya Str., 394006 Voronezh, Russian Federation \\ ${ }^{4}$ Air Force Academy named after Professor N.E. Zhukovsky and Yu.A. Gagarin (Voronezh) \\ 54a, Starykh Bol'shevikov Str., 394064 Voronezh, Russian Federation
}

\begin{abstract}
To solve a number of practical problems (oscillations of the supporting circuits in construction, the problem of plasma stabilization, stabilization of synthesized biologic chains, etc.), model systems are used which are based on the laws of motion of basic coupled oscillators and their chains. This article analyses the mathematical model of a system consisting of two inverted pendulums with a flexible joint (a spring). The system is controlled by a controller in the form of a vertical oscillation of the attachment point of one of the pendulums. The article presents a detailed study of the dynamics of the described mechanical system and determines the conditions for its stabilization. Stability zones were identified in the initial parameter space. The paper describes the evolution of the stability zones depending on the spring's stiffness. Spectra of solutions were obtained, showing that the motion of the system corresponds to almost periodic functions. It was established that unstable periodic regimes were present at the boundaries of the stability zones. The planes were determined that corresponded to the initial conditions which complied with the obtained periodic solutions. The key analytical results were obtained using the monodromy matrix. In our study we considered a situation when the system in the
\end{abstract}

Semenov Mikhail E.

e-mail: mkl150@mail.ru 


\section{М. Е. Семёнов, О. И. Канищева, М. А. Попов}

linear approximation is piecewise linear. For such a system the monodromy matrix can be given in explicit form. The article also presents the results of numerical experiments illustrating the dynamics of the system. It also demonstrates that when the parameters of the system are different from the initial values, the geometry of the stability zones also changes depending on the increase in the area of one of the zones. All figures illustrating the stability zones, evolution of the stability zones, solution spectra, pendulum motion graphs, and phase portraits were prepared using Wolfram Mathematica.

Keywords: inverted pendulum, coupled oscillators, stabilization, control, stability zones.

\section{CONFLICT OF INTEREST}

The authors declare the absence of obvious and potential conflicts of interest related to the publication of this article.

\section{REFERENCES}

1. Andronov A. A. Witt A. A. Khaikin S. E. Theory of oscillations. Moscow, Nauka. 1981. 568 p. (in Russian).

2. Bautin N. N. On the number of limit cycles appearing when the coefficients change from an equilibrium state of the focus or center type. Matematicheskii sbornik, 1952. 30 (72). 181-196. (in Russian).

3. Trubetskov D. I., Rozhnev A. G. Linear oscillations and waves. Moscow, Publishing House of Physical and Mathematical Literature, 2001. 416 p. (in Russian).

4. Magnus K. Oscillations: Introduction to research of oscillatory systems. Translation from German. Moscow, Mir, 1982. 304 p. (in Russian)

5. Osipov G. V. Synchronization in heterogeneous networks of oscillators. Nizhny Novgorod, 2014. 135 p. (in Russian).

6. Kolmogorov A. N. On the conservation of conditionally periodic motions for a small change in the Hamiltonian function, Doklady Akad. Nauk USSR, 1954. 98 (4). 527-530. (in Russian).

7. Butikov E. I. Stabilization of the inverted pendulum (60 years of Kapitza's pendulum). Computer tools in education, 2010. 5. 39-51. (in Russian).

8. Stephenson A. «On an induced stability». Phil. Mag, 1908. 15(233).

9. Kapitsa P. L. A pendulum with a vibrating suspension. UFN, 1951. 44. 7-20. (in Russian).

10. Kapitsa P. L. Dynamic stability of a pen- dulum with an oscillating point of suspension. JETP, 1951. 21. 588-597. (in Russian).

11. Nelepin R. A. Research methods for nonlinear automatic control systems. Moscow, Nauka, 1979. 447 p. (in Russian).

12. Krasnoselsky M. A., Pokrovsky A. V. Systems with hysteresis. Moscow, Nauka. 1983. 271 p. (in Russian).

13. Pliss V. A. Nonlocal problems of the theory of oscillations. Moscow, Nauka, 1964. 367 p. (in Russian).

14. Merkin D. R. Introduction to the theory of stability of motion. Moscow, Nauka. 1987. 304 p. (in Russian).

15. Matveev M. G., Semenov M. E., Shevlyakova D. V., Kanishcheva O. I. Zone of Stability and Periodic Solutions of the Inverted Pendulum with Hysteretic Control. Mechatronics, Automation, Control, 2012. 11, 8-14. (in Russian).

16. Semenov M. E., Hatif Z., Reshetova O. O., Demchuk A. A., Meleshenko P. A. The model of the dynamics with the inverted pendulum hysteretic control. Proceedings of Voronezh State University. Series: Physics. Mathematics, 2016. 4. 165-177. (in Russian).

17. Semenov M. E., Matveev M. G., Lebedev G. N., Solovyev A. M. Stabilization of a Flexible Inverted Pendulum with the Hysteretic Properties. Mechatronics, Automation, Control, 2017. 8. 516-525 (in Russian). DOI: 10.17587/ mau.18.516-525

18. Ryazhskih V. I., Semenov M. E., Rukavicin A. G., Kanishcheva O. I., Demchuk A. A., Meleshenko P. A. Stabilization of inverted pendulum on a two-wheeled vehicle, Bulletin of the South Ural State University Series "Mathematics. Mechanics. Physics" 2017. V. 9, No. 3. P. 41-50 (in Russian). DOI: 10.14529/mmph170306

19. Semenov M. E., Solovyov A. M., Popov M. A. Stabilization of unstable objects: cou- 
pled oscillators. Trudy MAI, 2017. 93 (in Russian).

20. Solovyov A. M., Semenov M. E., Meleshenko P. A., Reshetova O. O., Popov M. A., Kabulova $E$. G. Hysteretic nonlinearity and unbounded solutions in oscillating systems, Procedia Engineering, 2017, 201, 549 555. DOI: 10.1016/j.proeng.2017.09.634

21. Semenov M. E., Solovev A. M., Popov M. A., Meleshenko P. A. Coupled inverted pendulums: stabilization problem. Archive Of Applied Mechanics, 2018. 88. 517-524.

22. Semenov M. E., Popov M. A., Kanishcheva O. I. Nonlinear Related System of Inverted
Pentulums Control. Journal of Siberian Federal University. Engineering \& Technologies, 2018. 11(3). 280-290 (in Russian). DOI: 10.17516/1999494X-0040

23. Neimark Yu. I., Kogan N. Ya., Savelyev V. P. Dynamic models of control theory. Moscow, Nauka, 1985. 400 p. (in Russian)

24. Krasnoselsky M. A. Nonlinear almost periodic oscillations. Moscow, Nauka, 1970. 304 p. (in Russian).

25. Miroshnik I. V. Theory of automatic control. Nonlinear and optimal systems. St. Petersburg, Piter, 2006. 272 p. (in Russian).

Semenov Mikhail E. - DSc in Physics and Mathematics, Professor, Leading research fellow, Federal Research Centre Geophysical Survey of Russia Academy of Sciences; Department of Digital Technology, Voronezh State University.

E-mail: mkl150@mail.ru

ORCID iD: https://orcid.org/0000-0002-3361-1102

Kanishcheva Olesya I. - PhD in Physics and Mathematics, Associate Professor, Department of Mathematics, Air Force Academy named after Professor N. E. Zhukovsky and Yu. A. Gagari.

E-mail: oleka_olesya@mail.ru

ORCID iD: https://orcid.org/0000-0002-1830-4091

Popov Mikhail A. - postgraduate student, Department of Applied Mathematics and Mechanics, Voronezh State Technical University.

E-mail: soeltic@gmail.com

ORCID iD: https://orcid.org/0000-0003-4336-8190 